\title{
Diseño de un modelo de evaluación para procesos de autoevaluación de las especialidades médicas de la Universidad de Costa Rica, desde un enfoque de derecho a la educación
}

\author{
Diana Arce Flores \\ Máster en Evaluación Educativa \\ dafarce@yahoo.com \\ Universidad de Costa Rica, Costa Rica \\ Recepción: 30-09-2016 / Aceptación: 15-12-2016
}

\section{Resumen}

El artículo trata acerca de la relevancia, de partir de un enfoque en derechos humanos, particularmente el derecho a la educación, para el diseño de un modelo para los procesos de autoevaluación de las especialidades médicas de la Universidad de Costa Rica. El modelo enfatiza cuatro características - y sus elementos relacionados - que resguardan el derecho a la educación: disponibilidad, accesibilidad, aceptabilidad y adaptabilidad. Posteriormente se plantea de qué manera, a partir de la realidad del contexto de las especialidades médicas, las cuatro tipologías mencionadas serán tomadas en consideración en el modelo de autoevaluación.

Palabras clave: derechos humanos, autoevaluación, derecho a la educación, modelo, especialidades médicas.

\begin{abstract}
The article deals with the relevance of starting from a human rights approach, in particu lar the right to education, for the design of a model for the self-evaluation processes of the medical specialties of the University of Costa Rica. The model emphasizes four char acteristics - and their related elements - that safeguard the right to education: availability, accessibility, acceptability and adaptability. Later, it is proposed how, based on the reality of the context of the medical specialties, the four typologies mentioned will be taken into account in the self-evaluation model.
\end{abstract}

Key Words: human rights, self-evaluation, right to education model, medical specialties. 


\section{Introducción}

La Educación Superior experimenta en la actualidad una serie de retos importantes que han surgido a raíz de diversas situaciones políticas, económicas, sociales y culturales de carácter mundial. Precisamente, Schugurensky (1998) citado por Alcantarán (s.f.), describe claramente algunos acontecimientos y sus repercusiones, como, por ejemplo: la globalización, la disminución del Estado benefactor, el carácter mercantilista de algunas universidades privadas, la prioridad en los factores económicos sobre los sociales y culturales; el análisis costo-beneficio, entre otros. (p. 2). El mismo autor señala que cada disciplina académica se ve obligada a "probar" su valor, de acuerdo con los intereses económicos que se plantean a nivel mundial.

En las últimas dos décadas del siglo $\mathrm{XX}$ y principios del siglo XXI, este nivel educativo ha tenido un incremento en su matrícula debido a la creación de diversas instituciones universitarias, al crecimiento de la población, a los jóvenes provenientes de entes secundarios con aspiraciones a un mejor salario en el futuro, a la diversificación de las ofertas académicas, a los acelerados avances de la ciencia y la tecnología, entre otras. En el ámbito costarricense, más de cincuenta universidades privadas y cinco públicas han graduado por encima de 43000 profesionales en los últimos tres años. (Woodbridge, 2016).

En Costa Rica, al igual que en otros países, empiezan a proliferarse muchas instituciones de Educación Superior, sobre todo las de carácter privado; esto conlleva cambios en la sociedad, no sólo vistos desde una perspectiva de calidad en la formación de las personas, sino abordadas desde la sobrepoblación de profesionales en la nación, debido a los volúmenes de estudiantes que se gradúan en tiempos, generalmente menores, que los de las universidades estatales (Pérez, 2005). En un inicio, las instituciones de enseñanza superior carecían de un ente regulador, hasta que se crea el Consejo Nacional de Enseñanza Superior Universitaria Privada (CONESUP) a partir de la Ley 6693 en 1981 en Costa Rica.

Por ello, a partir de la década de los años ochenta se enfatiza en la necesidad de hacer una revisión reflexiva de los procesos académicos universitarios, en donde la evaluación juega un papel protagonista para respaldar la rendición de cuentas, la transparencia, y los logros de cada institución de educación superior, en respuesta a sus visiones, misiones y políticas respectivas. Aunado a lo anterior, la constante búsqueda del mejoramiento y de la toma de decisiones se reconoce como accionares inherentes del quehacer universitario $y$ a su calidad académica.

Específicamente, la formación de profesionales en Medicina en Costa Rica inició en el extranjero, a finales del siglo XIX, con profesionales graduados sobre todo en Europa; $y$ para el año 1850 inició esta disciplina en la Universidad de Santo Tomás (Fuentes, 2001). Posteriormente, para 1960 abre sus puertas la Escuela de Medicina de la Universidad de Costa Rica, y en 1964 esta casa de estudios 
asume la responsabilidad de extender los títulos de Médico, Cirujano y de Especialista.

En 1966, ocurre un hito en la formación en Medicina, pues se establecen los primeros vínculos de la docencia con el Seguro Social de Costa Rica, lo que llevó a la creación del Centro de Desarrollo Estratégico de Investigación en Salud y Seguridad Social (CENDEISSS) en 1974.

En el ámbito de especialidades médicas, desde el año 1976, la Caja Costarricense de Seguro Social (CCSS) ${ }^{1}$ inició una relación de colaboración con la Universidad de Costa Rica $(\mathrm{UCR})^{2}$, para ofrecer formación de posgrado en especialidades médicas; inclusive para el año 2010 se firma entre ambas instituciones el Convenio Marco entre la CCSS y la UCR para el desarrollo y ejecución de programas de pregrado, grado y posgrado, además de otros programas de capacitación, formación y el apoyo de la universidad a la gestión de la CCSS.

Actualmente, el voto 2015-6840, emitido por la Sala Constitucional, declaró inconstitucional la omisión de la Caja Costarricense del Seguro Social de suscribir acuerdos con universidades privadas que se encuentren en capacidad de formar médicos especialistas. De esta forma, se le ordenó a la CCSS que procediera, a suscribir dichos acuerdos con universidades privadas que así lo requirieran, siempre que éstas regulen las condiciones para ofrecer un posgrado, según el ordenamiento jurídico vigente, bajo los mismos términos que negoció con la UCR, sin imponer requisitos distintos.

El acontecimiento descrito anteriormente y el hecho de que el país no tenga experiencia en evaluación o en acreditación de la educación de especialidades médicas, a nivel nacional, y de sus respectivos servicios, conlleva a una nebulosa en relación con la capacidad de enseñar o prestar los servicios médicos señalados, garantizando la calidad de las instituciones de enseñanza (Arce y Gómez, 1998); debido a esta situación es que se define uno de los propósitos de este artículo, el cual radica en describir la necesidad sentida, tanto por el Sistema de Estudios de Posgrado (SEP) de la UCR, así como de las especialidades médicas; por diseñar un modelo de autoevaluación para estas últimas.

Asociado a este objetivo general, se ha reflexionado acerca de la importancia de considerar, como eje transversal, la conceptualización de la educación como un derecho, ya que, particularmente, en la creación de modelos estandarizados, frecuentemente se deja de lado el carácter democratizador y dignificador de la educación.

1. La autora también se referirá a esta institución mediante sus siglas.

2. También se mencionarán las siglas UCR como sinónimo de Universidad de Costa Rica. 


\section{Desarrollo}

Marco conceptual de los derechos humanos: derecho a la educación

Es necesario partir de un marco conceptual que permita al lector comprender el enfoque de los derechos humanos; qué es lo se entiende a partir de estos ideales y, específicamente, lo referente al derecho a la educación; ya que es importante analizar porqué pareciera que se ha llegado a una coyuntura, en el ámbito educativo, en la cual cada quien "deja hacer" y “deja pasar", conllevando esto a un pragmatismo deshumanizado, en el que el contexto se torna poco solidario. (Serrano, 1996, p. 51).

No obstante, según el mismo autor, no todo está perdido, ya que el solo hecho de que exista una conciencia relacionada con ese vacío de la educación, en el cual se cae en procesos que pareciesen más autómatas que humanos; permite que se haga retrospectiva y que se recapacite acerca del camino hacia dónde se quiere continuar.

Uno de los cuestionamientos que se pone sobre la mesa son las razones por las cuales, aunque se lleven a cabo innumerables esfuerzos por avanzar en materia de derechos humanos; en educación, no se logran los cambios o transformaciones significativas que se esperan. Tal vez sea necesario retroceder aún más en la historia, y retomar de qué forma nacen los sistemas educativos, antes de hacer referencia al tema de derechos; ya que los mismos, desde su carácter institucional, son relativamente recientes, nacen respondiendo a los intereses económicos de la industrialización, en la cual se venden estereotipos y se busca homogenizar a los sistemas para que se obtengan productos en el menor tiempo y costo posible, sin atender las necesidades de los trabajadores. Por ello, es que a pesar de que se intenten democratizar los sistemas educativos es complejo, debido a que los mismos fueron creados bajo intenciones totalmente contrarias (mercantilistas).

Se considera entonces, transformar el paradigma del sistema educativo tradicional, a un sistema educativo más flexible, basado en derechos humanos, en el cual se enfatice en valores como "la paz, la democracia, la libertad, la tolerancia y el respecto a la diferencia.” (Serrano, 1996, p. 52). Por otro lado, pero vinculado al cumplimiento de los derechos humanos, se menciona que a partir de las barbaries que ha vivido la humanidad en diversos momentos de la historia (caos), como por ejemplo guerras mundiales; han surgido cosas buenas (orden), como la Declaración Universal de los Derechos Humanos. Maturana (2002) comparte el término de "red caórdica”, en la cual el caos y el orden conviven para dignificar la vida y darle valor al aprendizaje con un propósito multidimensional.

En la Declaración mencionada (1948) se hace hincapié en los derechos fundamentales de libertad, vida, trato digno y sin discriminación, personalidad jurídica, seguridad social, el trabajo, la educación, entre otros. En este mismo sentido, el Pacto Internacional de Derechos Económicos, Sociales y Culturales, 
ONU (1976), en su artículo 13, se refiere a la educación como un derecho y camino para el desarrollo de la personalidad y su dignidad, garantizando la participación activa y libre en la sociedad, a partir de una cultura de paz (p. 2). Asimismo, destaca a la educación como la primera herramienta en manos de los Estados para mejorar las condiciones de los seres humanos desde su infancia, además de ser el derecho fundamental para la realización de los otros derechos. (Comité de Derechos Económicos, Sociales y Culturales, 1999, p.1)

Delors et al (1999) señalan que el propósito de la educación es potenciar los talentos y capacidades de creación de las personas; así como que la escuela, en cualquiera de sus niveles, debe fomentar el crecimiento del gusto por aprender para la vida y con esto ofrece la posibilidad de atender las necesidades de un contexto inmediato en una aldea global, en donde se considera al desarrollo como un proceso, jamás como un producto.

Al respecto, Muñoz (2009) propone que,

El derecho humano a la educación se centra en la tutela y protección del aprendizaje, como factor vital, que rechaza una connotación eficientista, caracterizada por el derecho positivo, al igual que una connotación inmanentista y por lo tanto inmutable, que caracteriza al derecho natural. (p. 18)

Para hacer cumplir los precedentes factores, se señala al Estado como el principal responsable, pero todos los miembros de la sociedad deben comprometerse con la realización de los derechos y para poder lograrlo, es imprescindible conocer dichos derechos humanos, ya que, si no se reconocen, ¿para qué tenerlos? De la misma forma que Delors et al (1999), Muñoz (2009) defiende la participación democrática de la sociedad civil en los procesos de toma de decisiones para la implementación adecuada del derecho a la educación (p. 6).

Otros aportes relevantes al tema de derechos humanos se reflejan en los documentos del Comentario General 1 (CND), de la Convención sobre los Derechos del Niño, de las Naciones Unidas (2001), particularmente en su artículo 29, en donde se destaca la importancia y las funciones de una educación fundamentada en los derechos humanos. Se enfatiza en las incapacidades del Estado para promover una educación holística y equilibrada, en la cual se destaque la participación de las familias, de las comunidades, de la ciudadanía en general; en donde se garanticen las cuestiones óptimas (financieras, humanas, de formación) que permitan la igualdad de oportunidades, el respeto hacia la diversidad, la no discriminación, entre otras.

En relación con la diversidad, se considera necesario mencionar que cada persona, a pesar de sus creencias, valores, credo, etc., debe ser capaz de respetar a los demás, aunque no compartan sus mismas ideologías, debe siempre defender y luchar por sus pensamientos, pero sin desvalorizar las opiniones de otros, ya que todos tenemos los mismos derechos a 
expresar las ideas propias y sentimientos; esta concepción es llamada por algunos autores como el respecto por la "otredad." (Serrano, 1996, p. 52). Abogar por el diálogo es la alternativa que se busca, ya que una de las características del ser humano es esa diversidad de la que se habla.

Por su lado, la Declaración de Dakar (2000), plantea diversos objetivos a futuro (2015), en el marco de la educación como un derecho, tales como: extender la cobertura, mejorar la educación de la primera infancia, en particular, para las poblaciones desfavorecidas; aumentar el número de adultos alfabetizados, erradicar las disparidades entre géneros; favorecer aspectos cualitativos de la educación, conseguir mejores resultados en lectura, escritura, aritmética y otras competencias prácticas básicas.

En esta misma línea, la Declaración d'Incheon (2015) retoma los acuerdos establecidos en el Foro Mundial sobre la Educación Dakar (2000), y propone los siguientes objetivos globales: garantizar educación inclusiva, equitativa de calidad que promueva oportunidad de aprendizaje para todos; esta educación inclusiva incluye a todos los niveles, al acceso a una educación de calidad con al menos nueve años de obligatoriedad, respetando la equidad de género; fortaleciendo un aprendizaje permanente.

Con el objetivo de reflexionar acerca de los aspectos de la educación como un derecho, tales como los que se han mencionado anteriormente, para el diseño de una modelo para procesos de autoevaluación para las especialidades médicas de la UCR, es imprescindible mencionar algunas cualidades particulares de las mismas.

\section{Generalidades de las especialidades médi- cas de la Universidad de Costa Rica}

Es importante mencionar que el programa de especialidades médicas en Costa Rica se basa en una metodología sui géneris, la cual consiste en un programa profesional de posgrado que posee características diferentes de las maestrías y doctorados regulares. La especialidad médica es un grado académico de posgrado exclusivo de la medicina humana; en ella, el estudiante aprende haciendo mediante una supervisión estrecha del profesor. Algunas tienen como requisito de ingreso la Licenciatura en Medicina y Cirugía, otras requieren otra especialidad en un campo afín, además de las evaluaciones correspondientes.

Los contenidos de la especialidad médica se plantean a partir de un diagnóstico de necesidades y temas de formación; se toman como base programas de especialidades de otros países, de universidades reconocidas y de asociaciones médicas; adaptándose algunos temas particulares a la epidemiología local.

El Comité Director de cada especialidad médica es el encargado de realizar las revisiones de los planes de estudio. Las actualizaciones están fundamentadas en el criterio experto de especialistas y se revisan, en promedio, cada cinco años. En este proceso participan la Unidad de Posgrado (conformada por los 
docentes de la especialidad), la Comisión del Programa de Posgrado en especialidades médicas (PPEM), la Comisión Interinstitucional CCSS-UCR, el Consejo del Sistema de Estudios de Posgrados, la Oficina Técnica de Planificación de la Educación Superior y el Consejo Nacional de Rectores (CONARE).

Dichos reajustes no se basan solamente en los contenidos y destrezas; también se ha aumentado el tiempo de preparación de los médicos-estudiantes en una cantidad considerable de especialidades debido a su complejidad. Actualmente más del 95\% de las especialidades médicas ha renovado sus planes de estudio. Las mismas se desarrollan tanto en los hospitales nacionales y especializados, como en los regionales y en otras áreas de salud. Los docentes son funcionarios de la Caja Costarricense del Seguro Social y de la UCR (Jaramillo y Villegas, s.f.), y los médicos residentes fungen como estudiantes y trabajadores de la Caja Costarricense del Seguro Social a la vez.

Las especializaciones son necesarias ya que los procedimientos médicos se han vuelto más complejos y eficaces; estas especializaciones tienen un impacto en el nivel político, debido a que contar con profesionales mejor capacitados aumenta la competitividad del país; además, se da una promoción a la innovación y al conocimiento lo cual es un estímulo económico tanto para los profesionales como para la nación entera (Britnell, 2011).

La importancia de abordar la formación de especialistas médicos radica en poder garantizar la calidad y no solamente la cantidad re- ferida a la preparación del recurso humano. Al existir un gran número de médicos generales graduados, cada vez se incrementa más la demanda por formarse en posgrado, y llevar a cabo investigaciones que ayuden a tener una óptima comprensión del contexto en este ámbito de la salud.

\section{Consideraciones para el diseño de un mo- delo de autoevaluación desde una aproxi- mación crítica del derecho a la educación}

Stockmann (2009), señala que la evaluación, constituye una forma de valoración o emisión de un juicio sobre una circunstancia o un objeto, esto con base en informaciones que se posean acerca de éste; las mismas son recolectadas, valoradas y es a partir de esto que se emite un juicio con el fin de tomar la decisión más idónea para el proceso.

Según este razonamiento, el desarrollo de una evaluación, independientemente de su finalidad específica, se efectúa según un modelo general. En él, se pueden identificar tres pasos a seguir: recolección información, valoración y toma de decisiones.

De acuerdo con Vigo, Segrea, Vitalia, Sánchez, López, Pons y Ramona, (2014), la evaluación institucional surge como una medida para objetivar la calidad de la educación alcanzada. Para ello, se busca aplicar "una serie de categorías, estándares, indicadores y variables de calidad que logren valorar el desempeño real de las instituciones educativas" (p. 728).

Asociado a lo anterior, según los mismos autores, la autoevaluación es considerada como 
"un mecanismo movilizador de todas las fuerzas internas de un centro, en función de un objetivo estratégico: la mejora continua de la calidad en busca de la excelencia universitaria" (p. 729); lo cual requiere un alto grado de compromiso, autocrítica y de participación de cada uno de sus miembros vinculados.

La autoevaluación es una forma interna de evaluación, orientada al mejoramiento de la calidad. En este sentido, debe estar destinada a fortalecer la capacidad de gestión de la unidad y conducir a una planificación sistemática de acciones de mejoramiento y a un seguimiento de las mismas.

Otro proceso a considerar es la acreditación cuya definición propuesta, toma como base los conceptos abordados por el Sistema Nacional de Acreditación de la Educación Superior (SINAES), el cual señala que la autoevaluación es la primera etapa para una acreditación oficial, en la cual la comunidad universitaria mediante un autoestudio que comprende una reflexión participativa y activa, se plantea a sí misma como objeto de estudio, explorando, analizando, diagnosticando, verificando, describiendo y valorando su realidad en cada una de sus estructuras orgánica, académica y administrativa.

Identificados los conceptos anteriores, se explica que el proyecto para el diseño del modelo de autoevaluación surge a raíz de las necesidades expresadas por las mismas espe- cialidades médicas, con el sustento del Sistemas de Estudios de Posgrados, debido a que los procesos formativos de éstas no han sido evaluados de una forma técnica-sistemática. Los únicos estudios que fueron desarrollados datan de la época de los noventa, realizados por CONARE, los cuales fueron una aproximación valorativa del funcionamiento de las especialidades médicas, más que una evaluación de sus dimensiones y pautas académicas. Al no existir un modelo de autoevaluación de ellas, propio de la Universidad de Costa Rica, es que se decide crear uno que se encuentre vinculado estrechamente con las bases evaluativas de SINAES, ${ }^{3}$ con el fin de que las especialidades médicas puedan implementarlo previamente a una acreditación formal y normada.

Se parte, para el presente análisis, de cuatro características primordiales para hacer valer el derecho a la educación (disponibilidad, accesibilidad, aceptabilidad, y adaptabilidad), según la aplicación del Pacto Internacional de Derechos Económicos, Sociales y Culturales (1999), específicamente las observaciones generales del artículo 13: El derecho a la educación. En primer lugar, se inicia por describir en qué consiste cada una de esas tipologías, de acuerdo con los factores relacionados con la Educación Superior; segundo, se comentará acerca de cuál es la realidad de las especialidades médicas ligadas a esa característica en particular, y posteriormente, se determinará la manera en la cual dicho rasgo podría o no incluirse en la propuesta del diseño del modelo de autoevaluación, según su alcance.

\footnotetext{
3. El modelo para procesos de acreditación del SINAES no puede ser aplicado tal cual a las especialidades médicas, debido a que el mismo no contempla las particularidades de este programa de posgrado. El carácter innovador del proyecto radica en la construcción e instrumentalización de un nuevo modelo, totalmente contextualizado a ellas.
} 


\section{a. Disponibilidad}

\section{Descripción}

Deben existir suficiente cantidad de instituciones de enseñanza en el ámbito del Estado Parte, con las condiciones necesarias para su funcionamiento, las cuales incluyen el contexto de desarrollo en el que actúan; por ejemplo, edificios, instalaciones sanitarias para ambos sexos, agua potable, docentes calificados con salarios justos, materiales para la enseñanza, bibliotecas, servicios de informática y tecnológicos, entre otros (p. 3).

\section{Realidad de las especialidades médicas}

Las especialidades médicas son un posgrado cuya formación académica se lleva a cabo en las instalaciones de la Caja Costarricense del Seguro Social, por esta razón, la UCR no tiene mucha injerencia en lo que se refiera a las instalaciones de los hospitales. El Estado es quien se encarga de dotar a los centros de salud públicos del país, del equipo necesario para su funcionamiento. Dichos equipos y las instalaciones con las que cuentan los médicos y los residentes para ejercer su práctica profesional, no siempre son los más adecuados.

De acuerdo con el ejercicio de recopilación de la información que la investigadora ha llevado a cabo, se ha confirmado a través de los medios de comunicación que, actualmente existe una fuerte campaña en el país que busca desfavorecer el gran esfuerzo que hace la UCR por garantizar la calidad de sus médi- cos especialistas; la misma alude a que no se cuenta con la cantidad suficiente de doctores que satisfagan las necesidades de la sociedad costarricense, pero no se divulga que los estudios de necesidades que ha llevado a cabo el Centro de Desarrollo Estratégico de Investigación en Salud y Seguridad Social ${ }^{4}$, carecen de una explicación metodológica-científica explícita, y se basan en estadísticas que no se vinculan al $100 \%$ con la realidad nacional, sino con los requerimientos institucionales particulares. Tampoco se menciona el currículum oculto que guía estos señalamientos, el cual atañe desde compromisos políticos hasta intereses personales, por dar cabida a centros educativos privados, así como a especialistas médicos que provengan del extranjero.

Desde el Centro de Desarrollo Estratégico de Investigación en Salud y Seguridad Social se han hecho sugerencias, en el año 2016, de contratar recurso humano de otros países, pero no se tienen criterios claros acerca de cuáles serán los requisitos para llevar a cabo dichas "importaciones" de especialistas, y tampoco se hace una descripción de cómo funcionarían y cuáles serían los aportes de los centros educativos privados a los recursos del Estado.

De acuerdo con el conocimiento de causa, del Programa de Posgrado en especialidades médicas $^{5}$, sí se cuenta con un número adecuado de especialistas formados, el cual responde al perfil epidemiológico de la población. En este momento, dicho programa cuenta con 863 estudiantes activos (de todas las especialida-

4. Dichos estudios se encuentran en la página web: www.cendeisss.sa.cr.

5. Las cifras e información que se expone son basadas en las estadísticas de este programa de la UCR, presentación elaborada por Villarreal y Sánchez (2016) para el Rector de esta institución. 
des) y entre los años 2006-2015 ha graduado a 1751 médicos especialistas, con una deserción estudiantil de 82 residentes únicamente, por distintas causas, la mayor de ellas por bajo rendimiento académico (43 personas). Asimismo, se omite informar a la sociedad que más que una carencia de especialistas, lo que existe es una carencia de equipo, de recursos materiales y de infraestructura, tales como salas de operaciones y equipo tecnológico acorde con la especialidad, sobre todo en los hospitales de zonas rurales; así como recurso humano que de soporte a los médicos que son obligados a ir a "trabajar" a zonas alejadas.

El punto acá es que en Costa Rica se ha aprobado que las universidades privadas oferten especialidades médicas y se le ha hecho pensar al pueblo que no existen suficientes especialistas en el país, pero al contrario de esto, lo que se quiere rescatar es que existirá un exceso de oferta académica, para la cual la Caja Costarricense del Seguro Social, con sus recursos actuales, no se encuentra capacitada para formar la cantidad de médicos que provengan tanto de universidades públicas como privadas; estas últimas son instituciones con fines de lucro, las cuales cobran (en matrícula y por cuatrimestres) más del cuádruple de lo que cobra la UCR. Ésta es una realidad nacional, que afectará a las especialidades médicas en cuanto a la calidad de los profesionales que se vayan a formar, con diferencias sustantivas de conocimientos y prácticas, en los hospitales, por dicho exceso de oferta.
Desde otra perspectiva, en cuanto a "contar con docentes calificados y con salarios competitivos", se reconoce una debilidad por parte de la UCR en este aspecto. Es evidente que dichas jornadas no van de la mano con el tiempo que dedican los médicos especialistas a los residentes, pero sería insostenible para la Universidad el poder nombrar a un profesor de tiempo completo, por cada estudiante, debido a que para las especialidades médicas, la formación es mediante una relación de uno a uno, en donde cada médico supervisa las labores de un estudiante; razón por la cual se deben repartir las jornadas de tiempos docentes en pequeñas partes o mediante nombramientos ad-honorem (más del 53\%).

Asimismo, al ser los profesores funcionarios de la CCSS, tampoco tienen derecho a dedicar de sus horas laborales como médico (en las cuales debe estar atendiendo consulta), al mejoramiento de su práctica estrictamente docente; por ello, al consultarle a cuatro tipos de poblaciones (docentes, residentes, coordinadores y comité director) de las especialidades médicas, en más de once talleres de autoevaluación acerca de las razones por las cuáles los médicos decidían ejercer como educadores de la UCR, contestaron que era una cuestión de mística y de retribución a la sociedad, no un medio para obtener remuneraciones económicas coherentes con la labor docente. 
¿Cómo se aborda la "disponibilidad" desde el diseño del modelo?

Como parte del autoconocimiento y la autorregulación de cada especialidad médica, y para poder diseñar el modelo para procesos de autoevaluación, que respondan a su contexto, se han realizado diversos talleres participativos, en los cuales se han determinado ciertas fortalezas y oportunidades de mejora acerca del funcionamiento de las especialidades. Algunas de ellas son cuestiones que corresponden a la Caja Costarricense del Seguro Social; mientras que otras son responsabilidad de la UCR, por lo cual deben buscarse soluciones para garantizar la óptima gestión académica del Programa de Programa de Posgrado en especialidades médicas.

Es de conocimiento general que, múltiples de los desafíos a los que se enfrentan las instituciones educativas, se encuentran vinculadas con los aspectos económicos. La situación de los docentes de las especialidades médicas no se excluye de dichas dificultades financieras, pero a sabiendas de que estos factores monetarios no son lo que impulsan a los profesores de la UCR a fungir como tutores de los residentes, se han explorado algunas alternativas que logren mitigar esa falencia salarial, tales como: acentuar el sentido de pertenencia a la Universidad de Costa Rica, lo cual genere un arraigo por la institución; el reconocimiento a los docentes que sean altamente calificados por medio de un instrumento de evaluación del desempeño que se está construyendo; realización de una actividad de inicio de año, en la cual se galardone a docentes por sus servicios a la universidad; ofrecimiento de cursos, talleres y charlas gratuitas que coadyuven al mejoramiento de su práctica docente, entre otros.

Todas las iniciativas mencionadas serán tomadas en cuenta en el diseño del modelo, en la dimensión de población docente, en una categoría denominada "cuestión docente", la cual abordará las condiciones materiales, estructurales, económicas y sociales en las cuales el profesorado ha de desempeñar su trabajo (Torres, 2005).

\section{b. Accesibilidad}

\section{Descripción}

Las instituciones y los programas de enseñanza deben ser accesibles a todas las personas, sin discriminación alguna, en el entorno del Estado Parte. Dicha accesibilidad se encuentra compuesta de tres dimensiones que se entrelazan (p. 3):

1. No discriminación: la educación debe ser accesible para todos, sin discriminación por ninguno de los motivos "de raza, color, sexo, idioma, religión, opinión, política o de otra índole, origen nacional o social, posición económica, nacimiento o cualquier otra condición social" (Pacto Internacional de los Derechos Económicos, Sociales y Culturales, 1976, p. 2).

2. Accesibilidad material: la educación debe ser posible materialmente, es decir, ya sea por su ubicación geográfica razonable o por medio de la tecnología, como lo son los programas de educación a distancia. (p. 3) 
3. Accesibilidad económica: la educación debe estar al alcance de todas las personas, en cuanto al nivel universitario específicamente, se menciona que la misma "debe hacerse igualmente accesible a todos, sobre la base de la capacidad de cada uno, por cuanto medios sean apropiados, y en particular por la implantación progresiva de la enseñanza gratuita." (Pacto Internacional de los Derechos Económicos, Sociales y Culturales, 1976, p. 5). Se solicita a los Estados Parte que se vaya promoviendo la gratuidad de la educación superior paulatinamente. (p. 3)

\section{Realidad de las especialidades médicas}

Con respecto a la accesibilidad a la educación superior, la UCR cuenta con una serie de políticas que sostienen su postura referente a la no discriminación. Por ejemplo, en las Políticas para los años 2010-2014, se señala en el Eje 3, Cobertura y Equidad, dentro del punto 3.1.1 se determina que la UCR "promoverá un proceso de admisión que optimice la equidad en el acceso a la Institución, tanto en grado como en posgrado" (p. 7); además en el punto 3.1.2., se indica que se "propiciará servicios de orientación e información ágil y eficiente sobre trámites de ingreso, oportunidades educativas y servicios a la población candidata a ingresar a la Institución" (p. 7). Finalmente, en este documento se expone, en el punto 3.3.1., que se "procurará condiciones óptimas para la permanencia y la graduación del estudiantado, sin ningún tipo de discriminación" (p. 7).
Por su lado, las Políticas de la UCR para los años 2016-2020, proponen en su Eje 7, de Gestión universitaria, punto 7.3.1., referido al Bienestar y vida universitaria, que la institución "promoverá activamente el desarrollo de un entorno académico y laboral, libre de toda forma de violencia y de discriminación, así como estrategias de acción afirmativa, para superar condiciones de desigualdad y de exclusión social, tanto de la población docente estudiantil como del talento humano docente y administrativo." (p. 7).

Con respecto a la accesibilidad material, la UCR cuenta con distintas sedes que posibilitan la asequibilidad a las carreras, tales como: Occidente, Atlántico, Guanacaste, Caribe, Golfito y la sede interuniversitaria de Alajuela. A pesar de ello, en relación con programas de posgrado, la oferta académica es bastante limitada. En relación con las especialidades médicas específicamente, las mismas se imparten en los principales hospitales nacionales, ubicados en las provincias de San José, Cartago, Heredia, Puntarenas, Limón, Guanacaste y Alajuela, por lo que, en este sentido, no habría un problema geográfico tan marcado, aunque para poblaciones indígenas $\mathrm{u}$ otras que se encuentren realmente alejadas de las comunidades urbanas, sí sería un problema el poder acceder a dichos centros de salud.

La característica referida a la accesibilidad económica, ha sido una de las más garantes por parte de la UCR. La gratuidad de la educación superior ha sido parcialmente respetada, debido a que siempre existe algún tipo 
de cobro y no es tan común que la población estudiantil reciba apoyos económicos para materiales, vivienda, traslado, etc.; pero se rescata que este centro de educación superior se ha preocupado por establecer un sistema de becas normado y es una institución sin fines de lucro. En comparación con otras universidades, sobre todo privadas, la UCR es la menos costosa en este ámbito. Además, la Universidad ha establecido políticas para que los posgrados de financiamiento regular sigan privando sobre los de financiamiento complementario. A nivel de posgrado, en el entorno de las especialidades médicas, no existen becas permanentes, ya que los estudiantes, al ser al mismo tiempo trabajadores de la CCSS, son remunerados mensualmente por sus labores mediante un salario, por lo que pueden cubrir sus gastos académicos. Los montos becarios para pasantías, congresos o simposios, entre otros; sí existen.

¿Cómo se aborda la “accesibilidad” desde el diseño del modelo?

Esta tipología del derecho a la educación, se abordará en la dimensión de "Gestión académica y administrativa" del modelo de autoevaluación. En cuanto a lo administrativo, el Centro de Desarrollo Estratégico de Investigación en Salud y Seguridad Social es quien lleva la batuta en este aspecto, pero el Programa de Posgrado en especialidades médicas puede y debe mejorar su gestión a lo interno del posgrado.

A pesar de que la accesibilidad es una característica difícil de cumplir en su totalidad, la
UCR se ha preocupado de que, por lo menos, los procesos de admisibilidad sean transparentes y lo más técnicos posible; cuestión que podría verse sumamente amenazada, al estar a cargo cualquier otro ente, ya sea de carácter nacional o internacional, como lo es el caso que ya se ha tramitado para el examen de internado médico.

\section{c. Aceptabilidad}

\section{Descripción}

La forma, el fondo, los programas de estudio y los métodos, en este caso andragógicos de la Educación Superior, deben ser aceptables, es decir, pertinentes, adecuados culturalmente y de buena calidad para la población estudiantil. (p. 3). En el párrafo 1 del artículo 13 del Pacto Internacional de los Derechos Económicos, Sociales y Culturales (1976) se sostiene que la educación debe potenciar el desarrollo pleno de la personalidad humana y del sentido de su dignidad, fortalecer el respeto por los derechos humanos, la libertad, capacitar a todas las personas para participar en una sociedad libre, promover la comprensión, la tolerancia, la amistad entre: naciones, grupos de distintas etnias, religiones, etc.; y favorecer los accionares de la ONU en procura del mantenimiento de la paz. (p. 5)

\section{Realidad de las especialidades médicas}

Como parte de los Políticas Universitarias de los años 2016-2020, se incluye en su II. Eje: Excelencia Académica, categoría 2.3. Evaluación y desarrollo académico, punto 2.3.1., que 
la universidad "apoyará a todas las unidades académicas y los programas de posgrado para que implementen procesos de autoevaluación sobre la pertinencia, calidad y gestión de los planes de estudio...” (p. 2)

Asimismo, el artículo 6 del Reglamento del Programa de Posgrado en especialidades médicas, declara en su inciso E que estos posgrados deben "procurar la calidad académica de los estudios universitarios de posgrado en especialidades médicas, al igual que la idoneidad de los docentes e investigadores participantes." (p. 2)

A pesar de dichos reglamentos, el cumplimiento de estas declaratorias no se ha cumplido a cabalidad. El Programa de Posgrado en especialidades médicas es un programa de suma importancia, el cual tiene un altísimo impacto en la sociedad y no ha sido tomado en cuenta para procesos de evaluación durante largos períodos de tiempo, debido precisamente a sus particularidades. Los centros hospitalarios públicos son el mejor ambiente para aprender, debido al volumen y a la complejidad de los casos que se presentan en ellos. Los recursos tecnológicos y de infraestructura, como se ha comentado, podrían ser mejores; a pesar de ello, se considera que en los hospitales centrales se logran satisfacer, en gran medida, los intereses de los médicos residentes en cuanto a su práctica profesional.

Las estrategias de enseñanza-aprendizaje se encuentran basadas en el "aprender haciendo", razón por la cual el fuerte de estos posgrados es la práctica y no así, los cursos teóricos.
En los talleres que se han llevado a cabo con algunas de las especialidades médicas, los residentes claman por estas clases magistrales, en las que puedan actualizarse más y ser guiados por sus docentes en relación con distintas temáticas. Estas demandas se ven limitadas por la función dual de estudiante-funcionario, en la cual no se les brinda espacio suficiente a los alumnos para invertir más tiempo en lo académico, sino que lo que priva para la Caja Costarricense del Seguro Social es que se disminuyan las listas de espera, no que se dediquen a la formación exclusivamente académica; aunque, posteriormente, esto se vea reflejado en una mejor atención a los ciudadanos usuarios de los servicios de salud.

En lo referente al aspecto culturalmente adecuado a los contextos se desconoce el cómo este aspecto se ve reflejado en la formación de los especialistas. Se podría basar en el supuesto de que los médicos tanto docentes como residentes deben estar preparados para atender a las distintas poblaciones que llegan diariamente a las consultas, las cuales provienen de un sinnúmero de lugares (dentro y fuera del país), con distintas creencias religiosas y diversidad cultural; pero esto sería simplemente una hipótesis, no una afirmación.

El componente de los derechos humanos de esta característica también puede relacionarse específicamente con el derecho a la salud, a tener una atención médica de calidad, basada en la equidad y en la igualdad. Todas las personas que requieran de atención hospitalaria de emergencia, la recibirán sin impor- 
tar si son nacionales o no, si son aseguradas o no, si son hombres o mujeres, si son ricos o pobres, entre otras; el sistema de salud costarricense no discrimina en ese sentido; pero lamentablemente si se habla de padecimientos, enfermedades menos fulminantes, o del requerimiento de algún tipo de examen médico, no es un secreto que el derecho a recibir una atención oportuna, se ve totalmente violentado, como por ejemplo, en los casos que se brindan citas médicas para dentro de un par de años o más.

Los valores de comprensión, tolerancia, respeto, entre otros; se visualizan en el ambiente laboral y académico. Las relaciones interpersonales con los compañeros de trabajo, con otros médicos, con los pacientes, etc., al igual que en otros climas laborales, determinan la vivencia de dichos valores en la cotidianidad.

\section{¿Cómo aborda la "aceptabilidad" el diseño del modelo?}

Desde el modelo de autoevaluación que se pretende diseñar, esta característica es una de las que tiene más peso, debido a su relevancia para la formación de los profesionales de las especialidades médicas. La misma se abordará en la dimensión de "Proceso formativo", en la cual se enfatizará en la calidad de los procesos de enseñanza-aprendizaje, pero a su vez, tomará en cuenta el trato que tienen los médicos para con sus pacientes, haciendo corresponder los valores de respeto, tolerancia, libertad de expresión, solidaridad, entre otros.
A su vez, el modelo abarca criterios de calidad, los cuales se vinculan totalmente con la aceptabilidad. De acuerdo con Villaruel (2010), el derecho a la educación se relaciona directamente con el desarrollo de los países y la evaluación en general, es un mecanismo o herramienta para poder detectar la implementación de los criterios de calidad, tales como: pertinencia, eficiencia, coherencia, transparencia, entre otros. Dichos criterios serán claves, y cada especialidad médica deberá demostrar con las evidencias respectivas, el cumplimiento de ellos como parte de su calidad académica.

\section{d. Adaptabilidad}

\section{Descripción}

Es necesario que la educación sea flexible para que pueda adaptarse a las necesidades de los contextos (sociedad y comunidad), los cuales son dinámicos y se encuentran en constante transformación. Además, debe responder a las necesidades de los estudiantes en contextos culturales y sociales diversos. (p. 4)

\section{Realidad de las especialidades médicas}

En sus inicios, las especialidades médicas se encontraban un poco desorganizadas ya que las mismas surgen a raíz de las necesidades de la sociedad de manera empírica, por lo cual sus características no son similares al resto de los posgrados de la UCR. Su existencia es anterior a la de CONARE, debido a esto, no se encontraban reconocidas y reguladas por esta entidad, hasta hace poco tiempo. 
Actualmente, las especialidades médicas han logrado llevar a cabo mejoras paulatinas. El 95\% de ellas ha conseguido renovar su plan de estudios, a partir de la reflexión y análisis de las nuevas tendencias en medicina, las necesidades de la sociedad, el estudio de recientes patologías, el perfil epidemiológico de la población costarricense, temáticas innovadoras, entre otras.

A su vez, los trabajos finales de graduación de los estudiantes permiten que se amplíen contenidos pertinentes para las especialidades, aunque cabe destacar que los mismos, en ocasiones, no son lo suficientemente difundidos por el gremio médico. Además, actualmente el Consejo Nacional de Investigación en Salud (CONIS), creado en 2014 a partir de la Ley N9234 (Ley reguladora de Investigación Biomédica), así como sus comités locales, han desfavorecido, de cierta manera, las investigaciones en las que participan seres humanos, ya que la eficiencia de dichos comités no ha sido la esperada por la población estudiantil, por lo que pueden atrasar ciertos procesos investigativos, así como a rechazarlos, sin tener conocimientos exhaustivos en la materia para hacerlo.

Por otra parte, relacionado con la adaptabilidad, el Programa de Posgrado en especialidades médicas se ve limitado por la Caja Costarricense del Seguro Social en cuanto a la ampliación de los años de estudio en las especialidades médicas; por ejemplo, el que una especialidad pase de cuatro años de formación a cinco, es sumamente difícil, ya que para la CCSS eso tiene un costo económico; a su vez, la UCR debe organizar mejor sus propuestas y justificaciones vinculadas con aumentar en cierto tiempo determinado una especialidad médica, debido a que también ha sucedido que se aumenta un año de estudio, $y$ éste último carece de un contenido, o rotaciones formalmente organizadas. Se menciona esta situación, debido a que el factor de flexibilidad necesaria para la adaptación de los planes de estudio, en este sentido, se puede ver afectado por las disposiciones de la Caja Costarricense del Seguro Social y organización de la especialidad médica.

\section{¿Cómo aborda la "adaptabilidad” el diseño del modelo?}

En el diseño del modelo se tomará en cuenta la característica de adaptabilidad en su dimensión de "Proceso formativo", en la categoría de plan de estudios, en el punto específico de metodología de enseñanza-aprendizaje. Se solicitarán evidencias no sólo relacionadas con el plan de estudios "teórico", sino acerca del cómo el posgrado se proyecta hacia las comunidades más vulnerables, cuáles aportes hace en diferentes contextos sociales y de qué manera genera cambios o transformaciones en el ámbito de la salud.

\section{Conclusiones}

Si bien es cierto que los procesos de autoevaluación con miras a la acreditación buscan un mejoramiento de la educación, los mismos no garantizan este hecho. Kumar (2011), señala que la acreditación no es necesariamente 
garantía de calidad; sin embargo, ayuda a la declaración pública sobre la capacidad de la institución para ofrecer programas eficaces y servicios basados en los requisitos acordados con el sistema de acreditación.

Es a partir de las definiciones que se han descrito anteriormente, sobre evaluación y la finalidad que se persigue, que Sánchez (s.f.) sugiere el siguiente decálogo:

- La evaluación debe propiciar el desarrollo de las potencialidades del objeto o sujeto que está siendo estudiado.

- La evaluación es un proceso de formación, por lo que no se centra únicamente en la obtención de nueva información.

- La evaluación no implica rigor en los planteamientos técnicos y eficacia de los resultados.

- La evaluación no persigue solamente un cambio tanto en los aspectos cognoscitivos, también influye en los valores y actitudes que fundamentan los procesos, así como en las personas que participan del mismo.

- La evaluación no propicia únicamente el cambio teórico de una actitud sino la participación de todos para su mejora.

- La evaluación genera un servicio social, así como un servicio personal.

- La evaluación es proceso mediante el cual se obtienen resultados.

- La evaluación no conlleva únicamente una valoración diagnóstica de partida, sino un proceso de implementación.
Otro concepto, imprescindible para el diseño de un modelo de autoevaluación es el de calidad, en este caso para definir "calidad de la Educación Superior" es importante hacer la distinción entre calidad de la enseñanza en la educación superior y la calidad de la Educación Superior. El primer punto está centrado en el proceso de transformación de la persona, en este caso del estudiante se refiere al proceso de enseñanza-aprendizaje que busca prioritariamente según Guzmán (2011):

- Enseñar a los alumnos a analizar ideas y temas de manera crítica.

- Desarrollar en los estudiantes las habilidades intelectuales y de pensamiento.

- Enseñar a los alumnos a comprender principios y generalizaciones.

Cuando se habla de calidad de la Educación Superior, puede hacerse referencia a los diferentes aspectos que son señalados por Garbanzo (2007) los cuales se vinculan más al proceso de evaluación del posgrado, como unidad de análisis y tomando principios de la educación como un derecho. Estos aspectos son:

- Propuestas curriculares pertinentes con las demandas contextuales globales

- Nivel académico y disponibilidad del profesorado

- Acceso equitativo de la población estudiantil

- Sistemas de beneficios estudiantiles para las poblaciones menos favorecidas socioeconómicamente 
- Rendimiento académico estudiantil

- Caracterización de la población estudiantil

- Relación presupuesto e inversión social

- Procesos administrativos flexibles y dinámicos

- Articulación con la investigación de alto nivel y su pertinencia con la realidad

- Social cantidad y calidad de sus publicaciones

- Acceso y uso de las tecnologías de avanzada al servicio de la academia

- Enlaces de cooperación a escalas nacional e internacional

- Visión humanista en la formación profesional

En esta misma línea, se rescatan las ideas de Muñoz, Artega y Méndez (2015) acerca de que la calidad, aun entendida como "excelencia”, es una cualidad, es decir, una característica, propiedad o circunstancia que distingue a las personas o cosas.

Por otra parte, los avances en el campo de la medicina exigen la formación en diferentes especialidades, que respondan a las necesidades en salud de la población, a los avances tecnológicos, a los perfiles epidemiológicos, a la capacidad instalada en los hospitales (material y humana), entre otras. Asimismo, el Programa de Posgrado en especialidades médicas realiza un invaluable aporte a la investigación por medio de la práctica profesional y de los trabajos finales de graduación de los médicos residentes, que no se visualiza debido a la falta de documentación sistematizada, la cual la autoevaluación podría ayudar a generar.
$\mathrm{Al}$ abrirse la oferta académica a las universidades privadas, se vuelve aún más imperante que las especialidades médicas estatales se organicen, mejoren y que se tomen decisiones que favorezcan la optimización de la formación de los futuros especialistas del país, tomando como base el enfoque del derecho a la educación.

Por otra parte, las plazas clínicas que asigna la Caja Costarricense del Seguro Social para formar médicos especialistas, en ocasiones no son suficientes, o en otras oportunidades, se asigna un número deliberado, según las presiones políticas que dicta el gobierno en curso. A pesar de ello, de acuerdo con el trabajo de campo que ha realizado la investigadora, se han hecho diversos esfuerzos por parte de esta institución por ampliar los cupos para algunas especialidades. A raíz de los talleres de autorregulación que se han llevado a cabo con las especialidades médicas, las mismas señalan que dicha apertura de plazas clínicas, de ninguna manera, puede ir en detrimento de la calidad del posgrado; por ejemplo, aunque se otorguen veinte plazas para una especialidad, si únicamente dieciocho estudiantes aprueban el examen de ingreso, por ningún motivo se debe bajar la dificultad de la prueba para cubrir las plazas faltantes.

En el ámbito educativo es difícil respetar y desarrollar los derechos humanos en un sistema que fue creado y pensando para una visión mecanicista, además los docentes carecen de condiciones adecuadas para poder facilitar la construcción de conocimientos desde estos derechos. Se enfatiza en cuatro elementos bá- 
sicos que son necesarios de tomar en cuenta en el campo de la educación:

- El acceso: derecho a la educación.

- El currículum: no solamente acceso a las instituciones de Educación Superior, para este caso en específico, sino también en un currículum inspirado en los derechos humanos.

- La cuestión docente: clave en los procesos de enseñanza aprendizaje, en donde se busca dignificar la profesión, con los recursos materiales necesarios para ejercer su práctica profesional.

- La gobernanza de las instancias educativas: referido este elemento al cómo se administra la universidad y a cómo se imparten lecciones.

El Estado, en general, debería basarse en una ética de derechos humanos, los cuales no pueden seguir siendo principios abstractos que se encuentren en las leyes, ni a acuerdos políticos que asumen las naciones, sino que es imperante desarrollar políticas públicas; acuerdos internos de los Estados que se inspiren en los derechos humanos, que las leyes se piensen en aspectos más concretos, deben tener una forma de desarrollarse en la práctica cotidiana de las comunidades.

Las políticas públicas son los mecanismos para legitimar y asumir las obligaciones del Estado desde un entorno más delimitado, sin dichas políticas públicas, y según el criterio de la investigadora, sin indicadores tanto cuantitativos como cualitativos; se hace imposible avanzar en ese cumplimiento de los derechos humanos, particularmente el derecho a la educación.

Deben existir, acuerdos políticos tanto internacionales, como nacionales y locales, en los cuales se manifieste el compromiso por la implementación de los derechos humanos.

\section{Referencias}

Alcántara, A. (s.f.) Tendencias mundiales en la educacion superior: el papel de los organismos multilaterales.

Arce, (s.d) y Gómez, (s.d). (1998). Criterio sobre el Proyecto de Reglamento para la inspección y acreditación de la carrera de medicina y cirugía. Ministerio de Educación Pública de Costa Rica.

Asamblea General de las Naciones Unidas. (1948). Declaración Universal de

Derechos Humanos. Recuperado de: http://www.ohchr.org/EN/UDHR/Documents/UDHR_ Translations/spn.pdf

Britnell, M. (2011). The role of the 'specialist' in healthcare. Clinical Medicine, 11(4), 329-331. Recuperado de: http://www.clinmed.rcpjournal.org/content/11/4/329.full 
Delors, J. et al. (1999). La educación encierra un tesoro. UNESCO-Santillana, Paris-Madrid. Recuperado de: http://www.unesco.org/education/pdf/DELORS_S.PDF

Declaración d'Incheon. (2015). Educación 2030 y Marco de Acción hacia una educación inclusiva y equitativa de calidad y un aprendizaje a lo largo de la vida para todos. Recuperado de: http://www.unesco.org/new/fileadmin/MULTIMEDIA/FIELD/Santiago/pdf/ESP-Marco-deAccion-E2030-aprobado.pdf

Elliot, J. (1993). El cambio educativo desde la investigación-acción, Madrid: Morata.

Foro mundial sobre la educación Dakar. (2000). Marco de Acción de Educación para Todos. Senegal. Recuperado de: http://unesdoc.unesco.org/images/0012/001211/121147s.pdf

Fuentes, C. (2001). Apuntes de la formación de especialidades médicas en la CCSS. Revistas de Ciencias Administrativas y Financieras de la Seguridad Social. Vol.9 (2), ISSN: 1409- 1259. Recuperado de: http://www.scielo.sa.cr/scielo.php?pid=S1409-12592001000200006\&script=sci_arttext

Garbanzo, G. (2007). Calidad y equidad de la educación superior pública. Aspectos por considerar en su interpretación. Revista educación, 31(2), 11-27. ISSN: 0379-7082, 2007.

Jaramillo, J. (2000). Evaluación y Acreditación para control de calidad en Escuelas de Medicina y Servicios Médicos Hospitalarios. San José: Editorial Universidad de Costa Rica.

Jaramillo, O. y Villegas, V. (s.f). Origen y desarrollo del programa de posgrado en especialidades medicas y psicologia social. Recuperado de http://www.binasss.sa.cr/revistas/rccm/v5s1/art3.pdf.

Kumar A. (2011). Calidad y acreditación en la educación superior en Centroamérica. Revista Centroamericana de Administración Pública. Instituto Centroamericado de Administración Pública,60 (61), 29-57.

Maturana, H. (2002). Transforción en la convivencia. Santiago: DOLMES EDICIONES S.A.

Muñoz, V., Arteaga, T., Méndez, M.V. (2015). Un enfoque innovador de la calidad educativa en la agenda post-2015.

Muñoz, V. (2009). El mar entre la niebla. El camino de la educación hacia los derechos humanos. Recuperado de: http://www.budrich.de/budrich-intern/El-mar-entre-la-niebla.pdf

Naciones Unidas. (2001). Comentario General 1 (CDN). Recuperado de: https://www.unicef. org/ecuador/UNICEF-ObservacionesGeneralesDelComiteDeLosDerechosDelNino-WEB.pdf 
Naciones Unidas. (1976). Pacto Internacional de Derechos Económicos, Sociales y Culturales. Recuperado de: http://campusvirtual.uned.ac.cr/lms/mod/folder/view.php?id=829120

Pérez, M. (2005). Creación de la Unidad de Gestión, Evaluación y Seguimiento de Posgrados. San José: UCR.

Sánchez, E. (s. f). Las agencias de acreditación: un puente hacia la calidad en la enseñanza superior. Universidad de Sevilla, España.

Stockmann, R. (2009). Evaluación y desarrollo de la calidad: bases para una administración de la calidad orientada a impactos. San José, Costa Rica: Editorial UCR

Torres, R. M. (2005). 12 tesis para el cambio educativo. Recuperado de http://www.feyalegria. org/images/acrobat/12\%20tesis_7043.pdf

Universidad de Costa Rica. (2013). Plan estratégico institucional 2013-2017. Recuperado de: http://oplau.ucr.ac.cr/phocadownload/plan_estrategico/Plan_estrategico_institucional_2013-2017.pdf

Universitat d Valencia. (2007). La evaluación de los estudiantes en la educación superior. Apuntes de buenas prácticas. Valencia, España. Servei de FormacióPermanent: Universitat de Valencia.

Vigo, P., Segrea, J., León, B., López, T., Pons, J., y Ramona, C. (2014). Autoevaluación institucional: Una herramienta indispensable en la calidad de los procesos universitarios.Medisur, 12(5), 728-732. Woodbridge, J. (2016). La educación y sus retos. La Nación. Recuperado de: http://www.nacion.com/opinion/foros/educacion-retos_0_1542645741.html 